\title{
Time-dependent multiconfiguration self-consistent-field study on resonantly enhanced high-order harmonic generation from transition-metal elements
}

\author{
Imam S. Wahyutama, ${ }^{1, *}$ Takeshi Sato, ${ }^{1,2,3, \dagger}$ and Kenichi L. Ishikawa ${ }^{1,2,3,+}$ \\ ${ }^{1}$ Department of Nuclear Engineering and Management, Graduate School of Engineering, The University of Tokyo, \\ 7-3-1 Hongo, Bunkyo-ku, Tokyo 113-8656, Japan \\ ${ }^{2}$ Photon Science Center, Graduate School of Engineering, The University of Tokyo, 7-3-1 Hongo, Bunkyo-ku, Tokyo 113-8656, Japan \\ ${ }^{3}$ Research Institute for Photon Science and Laser Technology, The University of Tokyo, 7-3-1 Hongo, Bunkyo-ku, Tokyo 113-0033, Japan
}

(Received 19 March 2019; published 20 June 2019)

\begin{abstract}
We theoretically study high-harmonic generation (HHG) from transition-metal elements $\mathrm{Mn}$ and $\mathrm{Mn}^{+}$using full-dimensional, all-electron, first-principles simulations. The HHG spectra calculated with the time-dependent complete-active-space self-consistent-field (TD-CASSCF) and occupation-restricted multiple-active-space (TDORMAS) methods exhibit a prominent peak at $\sim 50 \mathrm{eV}$, successfully reproducing resonant enhancement observed in previous experiments [Opt. Express 20, 25239 (2012)]. Artificially freezing 3p orbitals in simulations results in its disappearance, which shows the essential role played by $3 p$ electrons in the resonant harmonics (RH). Further transition-resolved analysis unambiguously identifies constructively interfering $3 p-3 d$ ( $m=0, \pm 1$ ) giant resonance transitions as the origin of the $\mathrm{RH}$, as also implied by its position in the spectra. Time-frequency analysis indicates that the recolliding electron combines with the parent ion to form the upper state of the transitions. In addition, this study shows that the TD-CASSCF and TD-ORMAS methods can be applied to open-shell atoms with many unpaired inner electrons.
\end{abstract}

DOI: 10.1103/PhysRevA.99.063420

\section{INTRODUCTION}

High-intensity and ultrashort laser pulses have become an indispensable tool, both in scientific research and industrial applications, for studying or manipulating the properties of matter. High-harmonic generation (HHG) is one of the important research domains that have emerged thanks to the remarkable advancement of high-intensity ultrashort laser technologies. HHG is a nonperturbatively nonlinear optical process in which a fundamental strong laser field is converted into harmonics of very high orders upon interaction with atoms, molecules, and solids. The nature of the HHG process is closely intertwined with the electronic structure and dynamics of the generating medium. Hence, a variety of quantum scale phenomena have been successfully identified by devising specialized measurement techniques such as electronic structure detection [1], observation of Rabi flopping [2], multichannel interference [3], and spectroscopy of Cooper minimum [4,5].

High-harmonic $(\mathrm{HH})$ radiation is an excellent source of coherent XUV photons that can fit into a labroom [6-8]. A number of applications for studying atomic and material properties have been reported [1-5], demonstrating its potential

\footnotetext{
*iswahyutama@atto.t.u-tokyo.ac.jp

†sato@atto.t.u-tokyo.ac.jp

¥ishiken@n.t.u-tokyo.ac.jp
}

Published by the American Physical Society under the terms of the Creative Commons Attribution 4.0 International license. Further distribution of this work must maintain attribution to the author(s) and the published article's title, journal citation, and DOI. as a reliable tool for studying light-matter interaction in the attosecond timescale. Further increase in harmonic intensity is desirable to fully explore its areas of use.

It has frequently been reported that the use of transitionmetal plasma as a generating medium leads to resonant enhancement of a single or a few harmonics [9-16]. The resonant harmonics $(\mathrm{RH})$ lie close to the giant transition lines in the absorption or emission response of the elements [9], e.g., $27 \mathrm{eV}$ in Sn plasma [10,13,14,17], $20 \mathrm{eV}$ in In [9], and $50 \mathrm{eV}$ in $\mathrm{Mn}$ [12]. The RH generation would offer an attractive way to increase HHG yield around a certain photon energy. It might also serve as a new platform to explore multielectron dynamics in intense laser fields using $\mathrm{HHG}$, which is usually considered to be of a single-electron nature in most cases.

In this work, we theoretically investigate the resonant $\mathrm{HHG}$ process using $\mathrm{Mn}$ and its cation $\mathrm{Mn}^{+}$as target systems for the scrutiny of the underlying mechanism. The resonance in $\mathrm{HHG}$ spectra from $\mathrm{Mn}^{+}$was observed experimentally by Ganeev et al. [12], where the harmonic peak at around $50 \mathrm{eV}$ is enhanced by more than an order of magnitude relative to neighboring harmonics, and the $3 p-3 d$ resonance was suggested to be relevant, as implied by the peak position [12].

Prior to the present work, there have been theoretical efforts on RH from transition-metal elements over the past decade. Milošević in Refs. [18] and [19] studied the effect of coherent superposition in the initial state and found that a three-step process starting from an excited state but returning to the ground state exhibits an enhanced harmonic. In Ref. [20], the line shape of the resonant harmonic is discussed in terms of Fano line shape. A modeling of the autoionizing state is performed in Refs. [16,17,21,22] using a parametrized potential barrier. Milošević has also investigated 
the properties of resonant harmonics such as intensity and phase in Ref. [23]. Other reports of varying elaboration have also been published, see e.g., Refs. [24-26]. Most of the above-mentioned attempts use an effective model potential within the single-active-electron approximation. Although not targeted at transition-metal plasma, there have been pioneering works $[27,28]$ that examine possible multielectron effects. For example, Redkin and Ganeev [27] have simulated a fullerenelike model system using the multiconfiguration time-dependent Hartree-Fock (MCTDHF) method within the two-active-electron jelliumlike sphere approximation. Pabst and Santra [28] have studied the effect of the giant resonance in HHG from Xe using the time-dependent configuration interaction singles (TDCIS) approach [29].

In the present work, we do all-electron three-dimensional (3D) ab initio simulations based on the time-dependent multiconfiguration self-consistent-field (TD-MCSCF) methods [30], which describe the system wave function by the superposition of Slater determinants consisting of time-dependent spin-orbital functions. Specifically, we apply state-of-the-art implementation of the time-dependent complete-activespace self-consistent-field (TD-CASSCF) [31-34] and timedependent occupation-restricted multiple-active-space (TDORMAS) [35] methods, which classify spatial orbitals into doubly occupied core and correlated active orbitals.

Previously, these methods have been applied to either closed-shell systems or systems having a single unpaired valence electron [32,35-37]. Here we extend our methods to general open-shell atoms such as transition metals having many unpaired electrons ( 5 and 6 for $\mathrm{Mn}$ and $\mathrm{Mn}^{+}$, respectively) that can equally participate in the dynamics under strong laser fields. We successfully reproduce the RH at $\sim 50 \mathrm{eV}$, and unambiguously identify the $3 p-3 d$ giant resonance as its origin, by taking full advantage of TD-CASSCF and TD-ORMAS to analyze transition dynamics between different orbitals. Our results show that the three $3 p-3 d$ lines $(m=0, \pm 1)$ constructively interfere to form the RH peak.

This paper is organized in the following way. The overview of the two methods used for our simulations is given in Sec. II. The results are presented and discussed in Sec. III. Conclusions and future possibilities are given in Sec. IV. Hartree atomic units are used throughout unless otherwise noted.

\section{TD-CASSCF AND TD-ORMAS METHODS}

We consider an $N$-electron atom (or ion) with atomic number $Z$ irradiated by a laser field $E(t)$ linearly polarized along the $z$ axis. In the velocity gauge and within the dipole approximation, its dynamics is described by the time-dependent Schrödinger equation,

$$
\begin{aligned}
i \frac{\partial}{\partial t} \Psi(t)= & {\left[\sum_{i=1}^{N}\left(\frac{-\nabla_{i}^{2}}{2}-\frac{Z}{r_{i}}-i A(t) \frac{\partial}{\partial z_{i}}\right)\right.} \\
& \left.+\sum_{i=1}^{N} \sum_{j>i}^{N} \frac{1}{\left|\mathbf{r}_{i}-\mathbf{r}_{j}\right|}\right] \Psi(t),
\end{aligned}
$$

with $A(t)=-\int E(t) d t$ being the vector potential.
TABLE I. Experimental ionization potential $I_{p}$, barrier suppression intensity $I_{B S}$, and the ground-state configuration of $\mathrm{Mn}, \mathrm{Mn}^{+}$, and $\mathrm{Mn}^{2+}$

\begin{tabular}{lccc}
\hline \hline & $\mathrm{Mn}$ & $\mathrm{Mn}^{+}$ & $\mathrm{Mn}^{2+}$ \\
\hline$I_{p}{ }^{\mathrm{a}}$ & 7.43 & 15.64 & 33.67 \\
$I_{B S}{ }^{\mathrm{b}}$ & $1.2 \times 10^{13}$ & $2.4 \times 10^{14}$ & $5.2 \times 10^{15}$ \\
$\mathrm{GS}^{\mathrm{c}}$ & {$[\mathrm{Ar}] 4 s^{2} 3 d^{5}\left({ }^{6} S_{5 / 2}\right)$} & {$[\mathrm{Ar}] 4 s 3 d^{5}\left({ }^{7} S_{3}\right)$} & {$[\mathrm{Ar}] 3 d^{5}\left({ }^{6} S_{5 / 2}\right)$} \\
\hline \hline
\end{tabular}

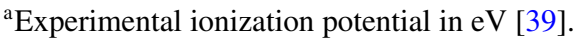

${ }^{\mathrm{b}}$ Barrier suppression intensity in $\mathrm{W} / \mathrm{cm}^{2}$.

${ }^{\mathrm{c}}$ Ground-state configuration [39].

In the TD-CASSCF and TD-ORMAS methods, we express the total wave function as

$$
\Psi(t)=\hat{A}\left[\Phi_{\mathrm{fc}} \Phi_{\mathrm{dc}}(t) \sum_{I} \Phi_{I}(t) C_{I}(t)\right],
$$

where $\hat{A}$ denotes the antisymmetrization operator, $\Phi_{\mathrm{fc}}$ and $\Phi_{\mathrm{dc}}$ the closed-shell determinants formed with $n_{\mathrm{fc}}$ timeindependent doubly occupied frozen-core and time-dependent doubly occupied $n_{\mathrm{dc}}$ dynamical-core orbitals, respectively, and $\left\{\Phi_{I}\right\}$ the determinants constructed from $n_{\mathrm{a}}$ active orbitals. Whereas in the TD-CASSCF method the active electrons are fully correlated among the active orbitals within prescribed numbers of up- and down-spin electrons, the TD-ORMAS method further subdivides the active orbitals into an arbitrary number of subgroups, specifying the minimum and maximum number of electrons accommodated in each subgroup.

TD-CASSCF and TD-ORMAS can systematically control the accuracy, until numerical convergence, through the number of orbitals and flexible orbital subspace decomposition. With TD-CASSCF, especially, we can take account of as many electron excitations as we want. Both methods are gauge invariant by virtue of the time-dependent variationally optimized orbitals [30,34]. Moreover, TD-CASSCF is size extensive while TD-ORMAS is not, which is not relevant to the atomic case. Whereas the computational cost of TD-CASSCF scales factorially with the number of active electrons, TDORMAS achieves a polynomial cost scaling, enabling even more efficient simulations.

We specifically consider $\mathrm{Mn}$ and $\mathrm{Mn}^{+}$in the present study, whose ionization potential, barrier suppression intensity $[16,38]$, and ground-state configuration are summarized in Table I. Orbital subspace decomposition used in this study is shown in Fig. 1. Note that at least 15 spatial orbitals are required for the correct spin multiplicities. TD-CASSCF simulations use 52 and 44 determinants for $\mathrm{Mn}$ and $\mathrm{Mn}^{+}$, respectively. In TD-ORMAS simulations, up to two-electron excitations from Active1 to Active 2 are allowed, which results in 86510 determinants for $\mathrm{Mn}$ and 66068 for $\mathrm{Mn}^{+}$.

The equations of motion (EOMs) describing the temporal evolution of the $\mathrm{CI}$ coefficients $\left\{C_{I}(t)\right\}$ and the orbitals $\left\{\psi_{p}(t)\right\}$ are derived on the basis of the time-dependent variational principle (TDVP) [40-43] and read

$$
\begin{gathered}
i \frac{d}{d t} C_{I}(t)=\sum_{J}\left\langle\Phi_{I}|\hat{H}-\hat{R}| \Phi_{J}\right\rangle, \\
i \frac{\partial}{\partial t}\left|\psi_{p}\right\rangle=\hat{h}\left|\psi_{p}\right\rangle+\hat{Q} \hat{F}\left|\psi_{p}\right\rangle+\sum_{q}\left|\psi_{q}\right\rangle R_{p}^{q},
\end{gathered}
$$




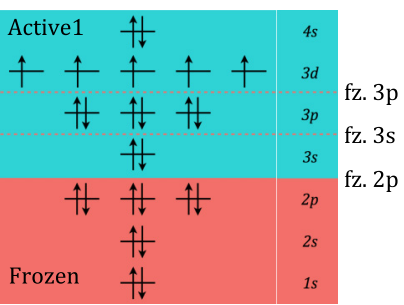

(a)

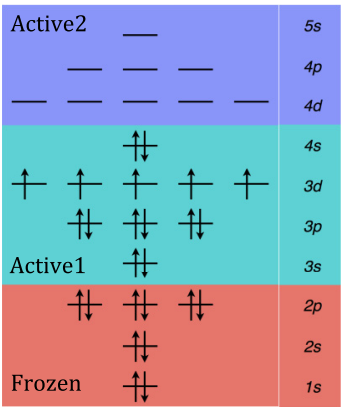

(c)

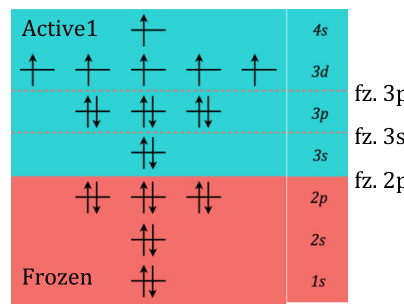

(b)

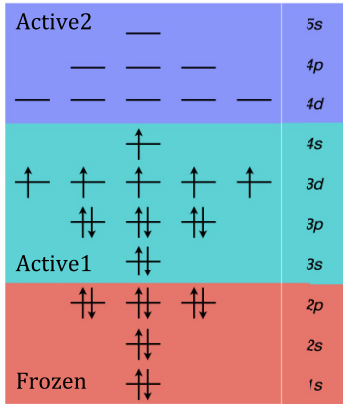

(d)
FIG. 1. Orbital diagram used for TD-CASSCF with 15 orbitals for (a) $\mathrm{Mn}$ and (b) $\mathrm{Mn}^{+}$. All the electron arrangements within Active 1 are allowed. The various frozen spaces are indicated by the dashed lines. For TD-ORMAS simulations with 24 orbitals, the schemes in (c) and (d) are used for $\mathrm{Mn}$ and $\mathrm{Mn}^{+}$, respectively, restricting up to two electrons in Active2.

where $\hat{H}$ denotes the total Hamiltonian, $\hat{h}$ the one-body Hamiltonian, and $\hat{Q}=1-\sum_{q}\left|\psi_{q}\right\rangle\left\langle\psi_{q}\right|$ the projector onto the orthogonal complement of the occupied orbital space. $\hat{F}$ is a nonlocal operator describing the contribution from the interelectronic Coulomb interaction, defined as

$$
\hat{F}\left|\psi_{p}\right\rangle=\sum_{o q s r}\left(D^{-1}\right)_{p}^{o} P_{o r}^{q s} \hat{W}_{s}^{r}\left|\psi_{q}\right\rangle,
$$

where $D$ and $P$ are the one- and two-electron reduced density matrices, and $\hat{W}_{s}^{r}$ is given, in the coordinate space, by

$$
W_{s}^{r}(\mathbf{r})=\int d \mathbf{r}^{\prime} \frac{\psi_{r}^{*}\left(\mathbf{r}^{\prime}\right) \psi_{s}\left(\mathbf{r}^{\prime}\right)}{\left|\mathbf{r}-\mathbf{r}^{\prime}\right|}
$$

The matrix element $R_{p}^{q}$ is given by

$$
R_{p}^{q}=i\left\langle\psi_{q} \mid \dot{\psi}_{p}\right\rangle-h_{p}^{q},
$$

with $h_{p}^{q}=\left\langle\psi_{q}|\hat{h}| \psi_{p}\right\rangle . R_{p}^{q}$, s within one orbital subspace (frozen core, dynamical core, and each subdivided active space) can be arbitrary Hermitian matrix elements, and in this paper, they are set to zero. On the other hand, the elements between different orbital subspaces are determined by the TDVP. Their concrete expressions are given in Ref. [35], where $i X_{p}^{q}=$ $R_{p}^{q}+h_{p}^{q}$ is used for working variables.

Our numerical implementation [32] employs a spherical harmonics expansion of orbitals with the radial coordinate discretized by a finite-element discrete variable representation (FEDVR) [44-47]. Specifically, each orbital is expanded with spherical harmonics whose largest angular momentum is 47. For TD-CASSCF simulations, the radial coordinate spanning up to 58.0 a.u. is divided into 16 finite elements, each of which contains 25 discrete variable representation (DVR) grid points, and an absorbing boundary using infiniterange exterior complex scaling (irECS) $[33,48]$ is placed at 46 a.u., which is almost twice as large as the quiver amplitude $\sim 26.4$ a.u. The time step is $\Delta t=0.0071$ a.u. For TDORMAS simulations on $\mathrm{Mn}\left(\mathrm{Mn}^{+}\right)$, the radial part spanning up to 242.0 (202.0) a.u. is divided into 62 (52) finite elements, each of which consists of 25 DVR grid points. A $\cos ^{1 / 4}$-shape mask function starting at 205.7 a.u. (160.0 a.u.) is used as an absorbing boundary. The time step is $\Delta t=0.0062(0.0106)$ a.u. The initial ground state is obtained through imaginarytime propagation of the EOMs using only 12 finite elements.

The Hartree-Fock energies $(-1149.866252$ a.u. for $\mathrm{Mn}$ and -1149.649383 a.u. for $\mathrm{Mn}^{+}$) perfectly match the values reported in Ref. [49]. For the CASSCF and ORMAS cases, electron correlation leads to the lowering of the ground-state energy. For example, the ORMAS method yields the groundstate energy of $\mathrm{Mn}$ to be -1150.0760 a.u. We confirm that the total orbital and spin angular momenta of the ground state match the term notations for both $\mathrm{Mn}\left({ }^{6} S_{5 / 2}\right)$ and $\mathrm{Mn}^{+}\left({ }^{7} S_{3}\right)$.

We calculate the HHG spectra as the magnitude squared of the Fourier transform of the dipole acceleration $a(t)$, defined as [32]

$$
\begin{aligned}
a(t) & =\sum_{i=1}^{N} \frac{d^{2}}{d t^{2}}\left\langle\Psi(t)\left|z_{i}\right| \Psi(t)\right\rangle \\
& =-Z \sum_{i=1}^{N}\left\langle\Psi(t)\left|\frac{z_{i}}{r_{i}^{3}}\right| \Psi(t)\right\rangle-N E(t)+\Delta\left(\dot{p}_{z}\right) .
\end{aligned}
$$

Here the additional term $\Delta\left(\dot{p}_{z}\right)$ accounts for the correction to the Ehrenfest formula in the presence of frozen core orbitals. Its explicit expression is found in Ref. [32].

\section{RESULTS AND DISCUSSIONS}

\section{A. Resonant high-harmonic emission from $\mathrm{Mn}$ and $\mathrm{Mn}^{+}$}

Let us first examine if our simulations reproduce the resonant harmonics in the $\mathrm{HH}$ response of $\mathrm{Mn}$ and $\mathrm{Mn}^{+}$. The harmonic spectra from $\mathrm{Mn}$ and $\mathrm{Mn}^{+}$obtained with the TDCASSCF method for a fundamental laser field with $770 \mathrm{~nm}$ central wavelength, $3 \times 10^{14} \mathrm{~W} / \mathrm{cm}^{2}$ peak intensity, and footto-foot four-cycle $\sin ^{2}$ pulse shape are shown in Figs. 2(a) and 2(b) (blue solid, marked as "fz. 2p"), respectively. The results of the MCTDHF simulations, in which all the 15 orbitals in Figs. 1(a) and 1(b) are treated as active, are also shown (green thick solid curves). The perfect overlap of the results by the two methods indicates numerical convergence for this number of orbitals. The results of the TD-ORMAS simulations on $\mathrm{Mn}$ and $\mathrm{Mn}^{+}$are plotted in Fig. 3 (dark green) for the same laser parameters as in Fig. 2. In both Figs. 2 and 3 , we clearly see an RH slightly above $50 \mathrm{eV}$, substantially enhanced in comparison with neighboring harmonics, for both $\mathrm{Mn}$ and $\mathrm{Mn}^{+}$, whose position is in excellent agreement with the experimental value $(\sim 50 \mathrm{eV} \mathrm{[12])}$.

We have also calculated $\mathrm{HH}$ spectra for $1333 \mathrm{~nm}$ wavelength and $10^{14} \mathrm{~W} / \mathrm{cm}^{2}$ peak intensity with the TD-ORMAS method (Fig. 4), while keeping the ponderomotive energy unchanged. In spite of the substantial difference in laser parameters, we can see that the resonant harmonic peak remains 


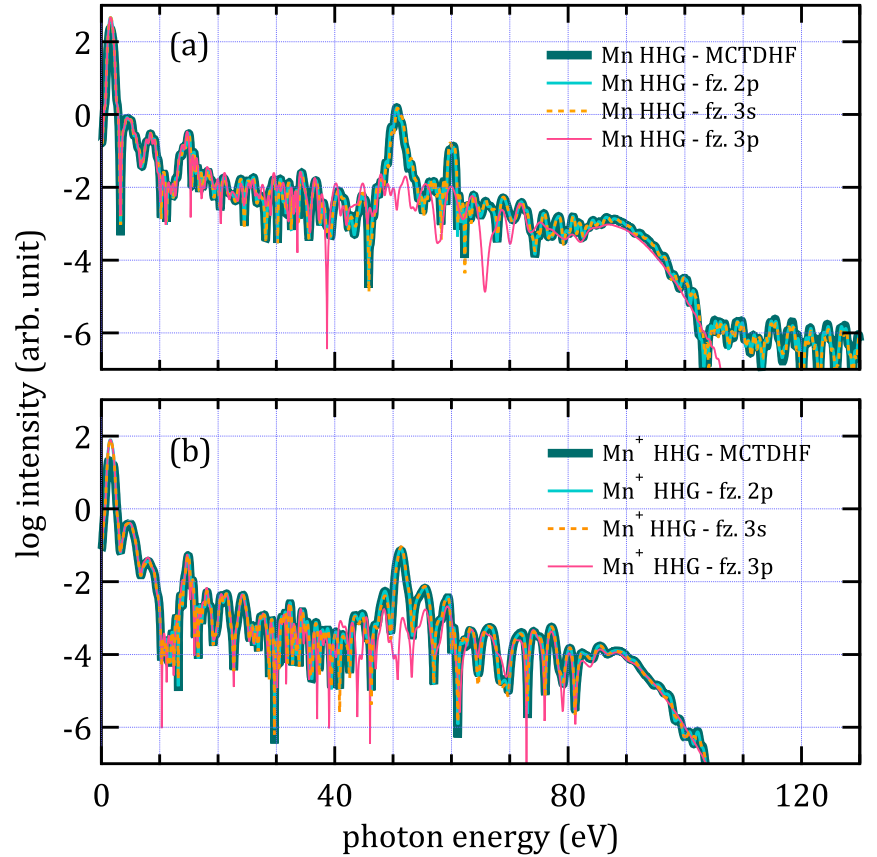

FIG. 2. HHG spectra calculated with the TD-CASSCF method for several frozen-core settings shown in Figs. 1(a) and 1(b) for (a) $\mathrm{Mn}$ and (b) $\mathrm{Mn}^{+}$. The results of the MCTDHF simulations, in which all 15 orbitals in Figs. 1(a) and 1(b) are treated as active, are also shown.

at $\sim 50 \mathrm{eV}$. Thus, the $\mathrm{RH}$ position is governed by atomic properties rather than laser parameters.

We then make use of the flexibility in orbital subspace decomposition to find out which orbitals contribute to RH. We have performed TD-CASSCF simulations by varying the boundary between the active and frozen spaces in Fig. 1. Whereas freezing $3 s$ virtually does not change the spectrum, freezing up to $3 p$ leads to the disappearance of the RH peak (Fig. 2). This indicates that the appearance of the enhanced peak involves the dynamics of $3 p$ electrons.

In Fig. 5 are shown the (single-photon) excitation spectra of $\mathrm{Mn}, \mathrm{Mn}^{+}$, and $\mathrm{Mn}^{2+}$, obtained as a Fourier transform of the dipole response to a quasi-delta-function pulse with the field being finite at three time steps $\left(10^{8} \mathrm{~W} / \mathrm{cm}^{2}\right.$ peak intensity). The overall spectral features obtained by both methods agree with each other. Especially, we see a strong excitation line at $\sim 50 \mathrm{eV}$ in all cases, which reproduces the position of the well-known 3p-3d giant resonance line [52,53] and coincides with the RH in the HHG spectra. These observations strongly suggest that the RH originates from the $3 p-3 d$ resonance line, as also implied experimentally [12].

Before ending this section, let us briefly discuss the cutoff energies. The arrows in Fig. 3 mark the cutoff positions $E_{c}$ expected from the cutoff law $E_{c}=I_{p}+3.17 U_{p}$, with $U_{p}$ being the ponderomotive energy. Even if the simulation starts from $\mathrm{Mn}$ or $\mathrm{Mn}^{+}$, the $\mathrm{HHG}$ spectra (thick solid dark green) extend further up to the cutoff corresponding to $\mathrm{Mn}^{2+}$. Indeed, as expected from the barrier suppression intensity (Table I) and confirmed by Fig. 6 showing the temporal variation of the fraction of each species, $\mathrm{Mn}$ is mostly ionized in the early stage, and $\mathrm{Mn}^{+}$is further substantially ionized to $\mathrm{Mn}^{2+}$. The comparison between Fig. 6 and the time-frequency

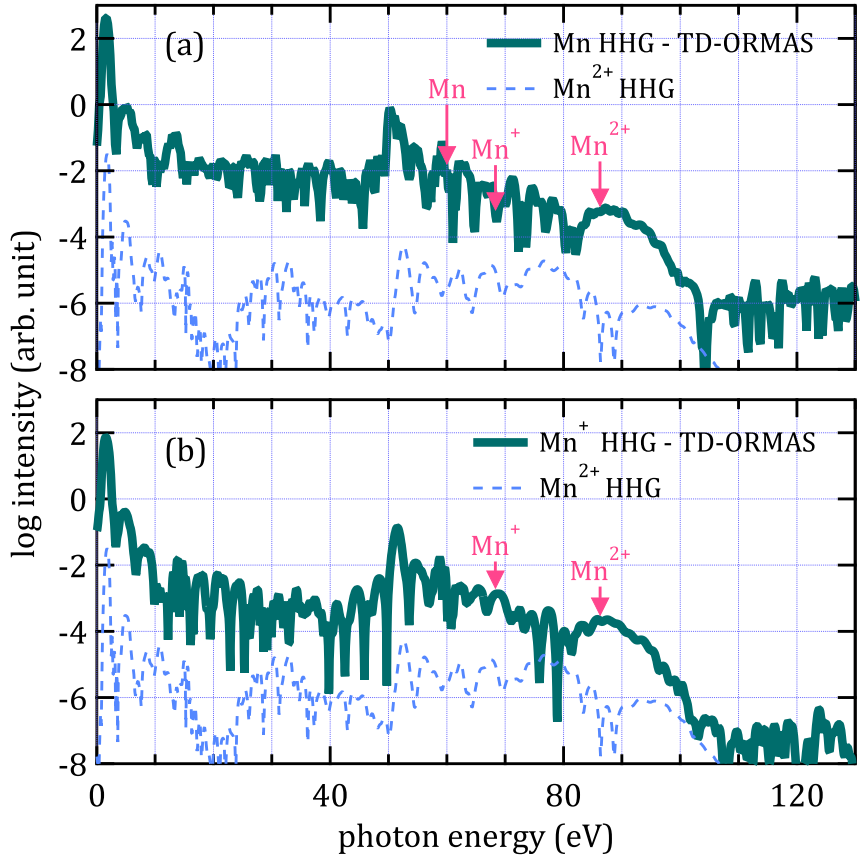

FIG. 3. HHG spectra calculated with the TD-ORMAS method for (a) $\mathrm{Mn}$ and (b) $\mathrm{Mn}^{+}$(dark green). The vertical arrows mark the cutoff positions expected from the three-step model [50,51]. The HHG spectrum from $\mathrm{Mn}^{2+}$ obtained using TD-CASSCF with 14 orbitals (the same orbital diagram as Fig. 1(b) except that $4 s$ orbital is absent) is also plotted (dashed blue).

structure of HHG shown in Fig. 7 also indicates that the higher plateau appears after the production of $\mathrm{Mn}^{2+}$. If we start simulations from $\mathrm{Mn}^{2+}$ as the initial state, however, the harmonic intensity becomes lower by 2 or more orders of

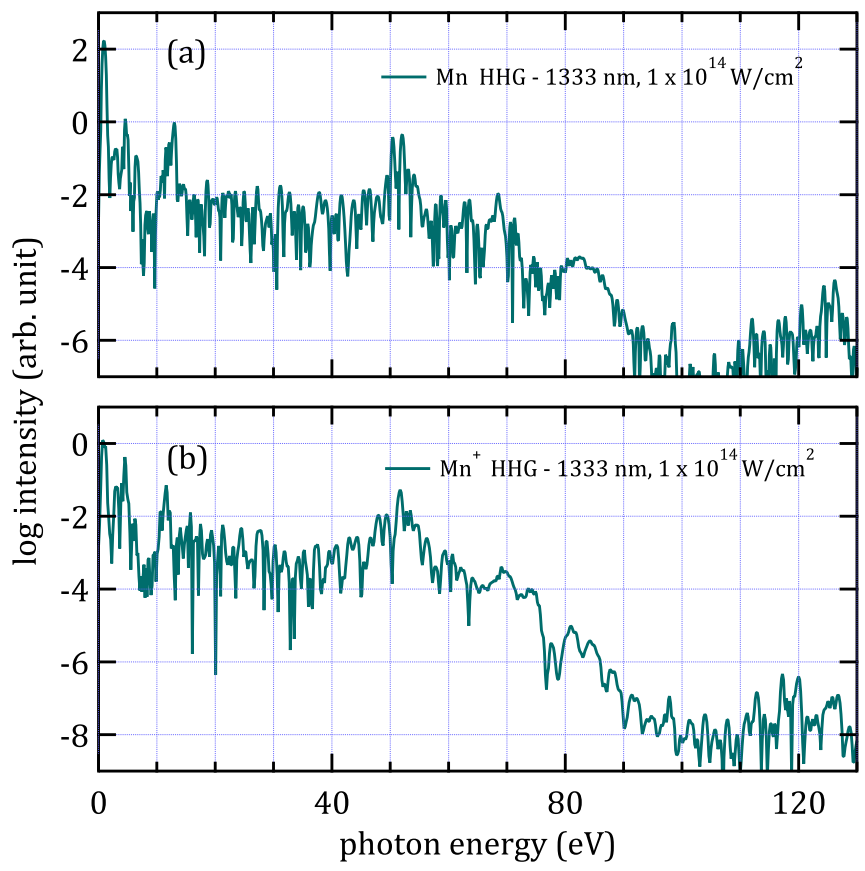

FIG. 4. HHG spectra from (a) $\mathrm{Mn}$ and (b) $\mathrm{Mn}^{+}$calculated with the TD-ORMAS method for $1333-\mathrm{nm}$ wavelength and $10^{14} \mathrm{~W} / \mathrm{cm}^{2}$ peak intensity. 


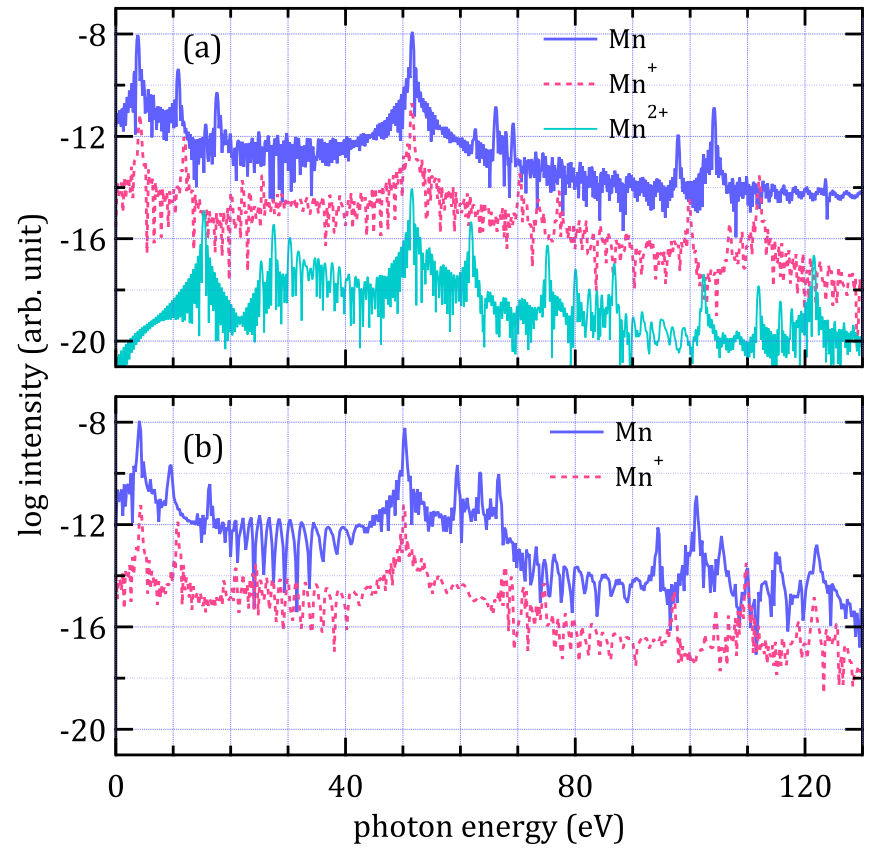

FIG. 5. Photoexcitation spectra calculated with (a) TD-CASSCF and (b) TD-ORMAS. The same orbital subspace decomposition used for the $\mathrm{Mn}^{2+} \mathrm{HH}$ spectrum in Fig. 3 is used for its photoexcitation spectrum in (a). The spectra of $\mathrm{Mn}^{+}$and $\mathrm{Mn}^{2+}$ are multiplied by $10^{-3}$ and $10^{-6}$, respectively, for better visibility. The excitation spectrum of $\mathrm{Mn}^{2+}$ could not be stably calculated with the TD-ORMAS method.

magnitude (Fig. 3, dashed blue line), as expected from the large ionization energy (Table I). The harmonic response of $\mathrm{Mn}^{2+}$ is enhanced most likely through laser-induced electron recollision [36,37,54], where tunneling ionization of $\mathrm{Mn}^{2+}$ is assisted by the Coulomb force from the recolliding electron which is ejected from $\mathrm{Mn}^{+}$.

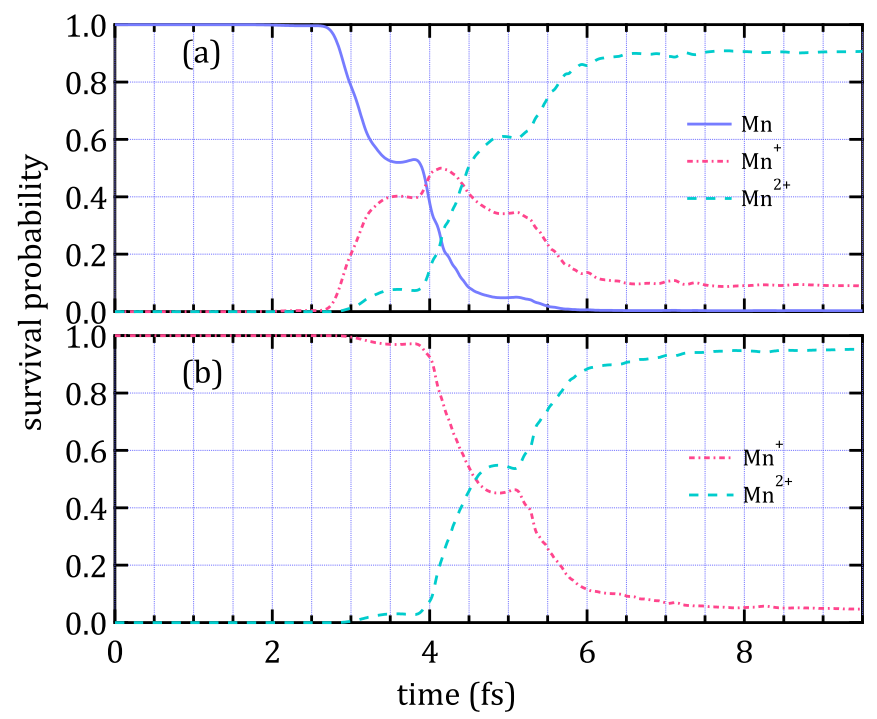

FIG. 6. Temporal evolution of the survival probabilities of Mn, $\mathrm{Mn}^{+}$, and $\mathrm{Mn}^{2+}$ during the TD-ORMAS simulations starting from (a) $\mathrm{Mn}$ and (b) $\mathrm{Mn}^{+}$under the conditions used in Fig. 3.

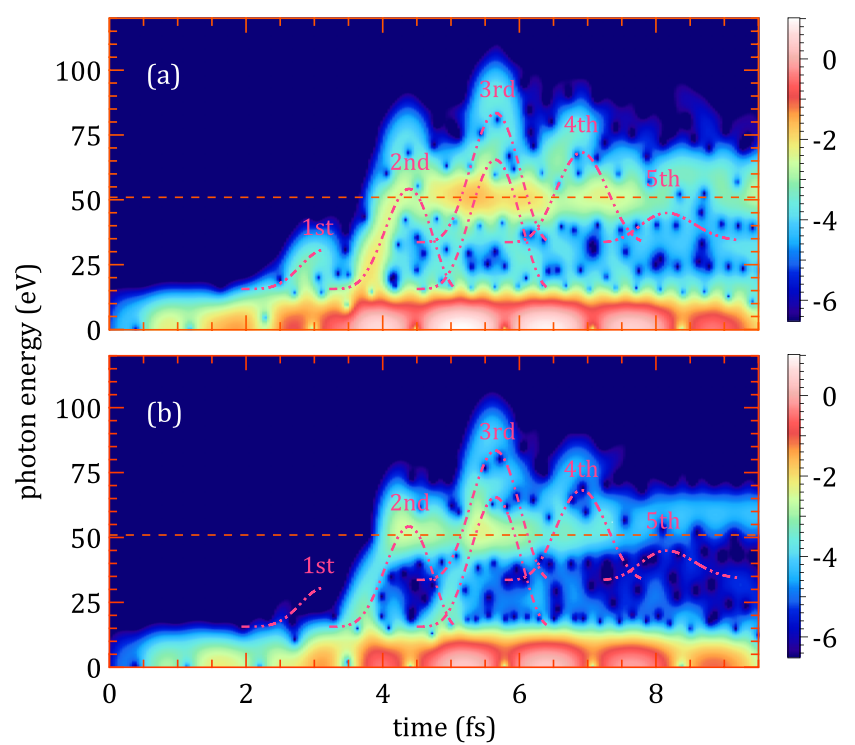

FIG. 7. The time-frequency spectrograms of HHG from (a) Mn and (b) $\mathrm{Mn}^{+}$obtained by Gabor transforming the TD-ORMAS results (Fig. 3) with a time window size of 7.5 a.u. The lower and upper groups of recombination energy (sum of the kinetic energy of the returning electron and the ionization potential) curves are for $\mathrm{Mn}^{+}$and $\mathrm{Mn}^{2+}$, respectively.

\section{B. Transition-resolved analysis}

The results in the previous section motivate us to analyze contributions from individual transition lines. For the transition-resolved analysis, let us rewrite the dipole acceleration Eq. (8) as

$$
\begin{aligned}
a(t)= & \sum_{m, n}\langle m|\hat{f}| n\rangle\langle n|D(t)| m\rangle-N E(t)+\Delta\left(\dot{p}_{z}\right) \\
= & 2 \sum_{n} \sum_{m>n} \operatorname{Re}\{\langle m|\hat{f}| n\rangle\langle n|D(t)| m\rangle\}, \\
& -N E(t)+\Delta\left(\dot{p}_{z}\right),
\end{aligned}
$$

where $\hat{f}=-Z\left(z / r^{3}\right)$, and $\{|n\rangle\}$ denotes the initial orbitals, obtained through imaginary-time relaxation. Since each initial orbital has a definite parity, the terms for $m=n$ vanish. Then we can view

$$
\alpha(m, n, t)=2 \operatorname{Re}\{\langle m|\hat{f}| n\rangle\langle n|D(t)| m\rangle\}
$$

as a contribution from a transition between orbital pair $m$ and $n$ to the dipole acceleration. With the orbitals used in TDORMAS simulations [Figs. 1(c) and 1(d)], we can identify the following 18 transitions, satisfying the selection rule:

$$
\begin{array}{rlrl}
3 s & \leftrightarrow 3 p_{0} & 3 s & \leftrightarrow 4 p_{0} \\
3 p_{0, \pm 1} & \leftrightarrow 3 d_{0, \pm 1} & 3 p_{0, \pm 1} & \leftrightarrow 4 d_{0, \pm 1} \\
3 p_{0} & \leftrightarrow 4 s & 3 p_{0} & \leftrightarrow 5 s \\
4 s & \leftrightarrow 4 p_{0} & 3 d_{0, \pm 1} & \leftrightarrow 4 p_{0, \pm 1} \\
4 p_{0, \pm 1} & \leftrightarrow 4 d_{0, \pm 1} & 4 p_{0} & \leftrightarrow 5 s,
\end{array}
$$

where the subscripts denote the magnetic quantum numbers. We have calculated the power spectrum of each transition line as the magnitude squared of Fourier transform of $\alpha(m, n, t)$. 


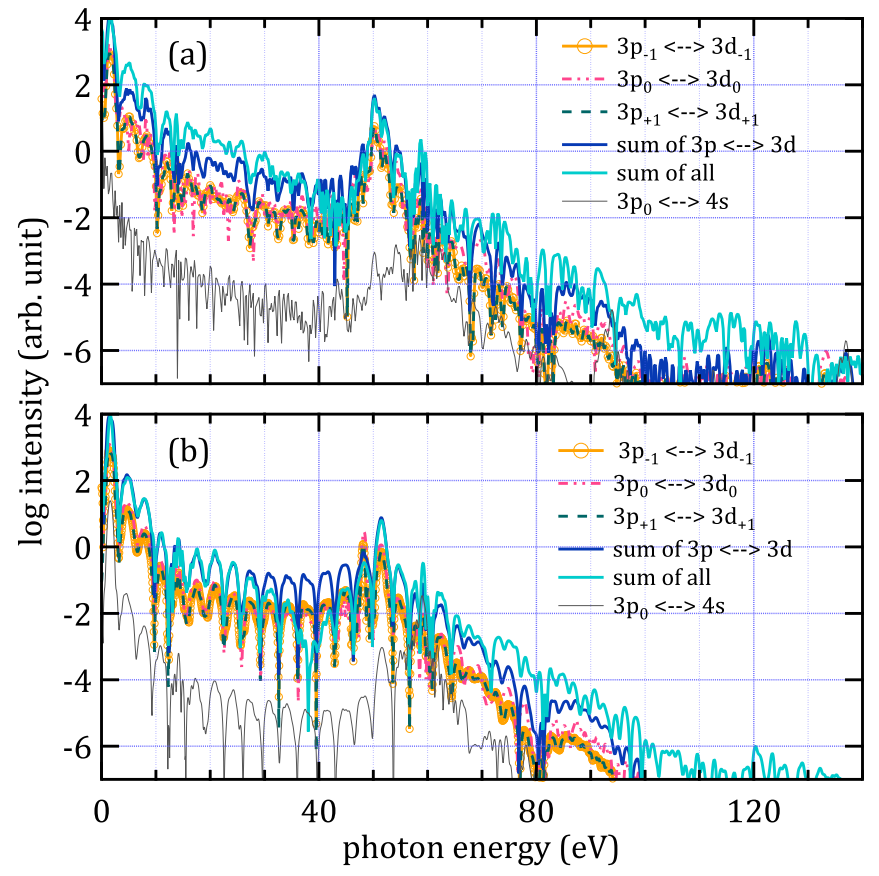

FIG. 8. Power spectra of each $3 p \leftrightarrow 3 d$ transition, $3 p \leftrightarrow 4 s$, the sum of the three $3 p \leftrightarrow 3 d$ transition components, as well as the sum of all the 18 transitions listed in Eq. (11) for (a) Mn and (b) $\mathrm{Mn}^{+}$.

The spectrum of each of $3 p_{0, \pm 1} \leftrightarrow 3 d_{0, \pm 1}$, the contribution of their sum, and the total spectrum of all the lines listed in Eq. (11) are presented in Fig. 8 for $\mathrm{Mn}$ and $\mathrm{Mn}^{+}$. The sum contribution of $3 p_{0, \pm 1} \leftrightarrow 3 d_{0, \pm 1}$ (solid dark blue) has a clear peak at $\sim 50 \mathrm{eV}$ and dominates the total contribution in that photon energy region. The other transitions involving $3 p$, such as $3 p_{0} \leftrightarrow 4 s$ (thin gray), are by orders of magnitude weaker. Moreover, the position and form of the peak agree very well with those of the RH in the HHG spectra shown in Fig. 3. It should also be noticed that the sum spectrum of the three $3 p_{0, \pm 1} \leftrightarrow 3 d_{0, \pm 1}$ lines is approximately 1 order of magnitude stronger than the contribution of each transition. These observations unambiguously establish that the resonant harmonic at $\sim 50 \mathrm{eV}$, experimentally discovered [12] and numerically reproduced by our ab initio simulations, is driven by a constructive interference of electron dynamics occurring between $3 p$ and $3 d$ orbitals.

Superposed on the spectrograms in Fig. 7, we plot, as a function of time, the recombination energy, defined as the sum of the kinetic energy of the returning electron and the ionization potential, or, harmonic photon energy expected from the three-step model [50,51]. We see that RH photons are emitted mainly when the recombination energy is $\sim 50 \mathrm{eV}$, where the recolliding electron has to be recaptured by the parent ion in order to induce a $3 p \rightarrow 3 d$ transition. This is consistent with recombination to autoionizing states (the upper states of the $3 p$ - $3 d$ giant resonance lines in the present case), proposed in Refs. [16,17,21,22]. Whereas these studies used the single-active-electron approximation with a model potential barrier, our all-electron $a b$ initio simulations support this process as a mechanism of $\mathrm{RH}$ generation from $\mathrm{Mn}$ and $\mathrm{Mn}^{+}$.

\section{CONCLUSIONS}

We have applied the TD-CASSCF and TD-ORMAS methods to open-shell elements to study resonant enhancement in high-harmonic generation from transition-metal elements $\mathrm{Mn}$ and $\mathrm{Mn}^{+}$. Our simulations have successfully reproduced the presence and position $(\sim 50 \mathrm{eV})$ of the experimentally observed resonant harmonics [12]. While its position suggests the relevance with the $3 p-3 d$ giant resonance lines, we have performed a series of analyses to unambiguously verify it. First, we have taken advantage of flexibility in orbital subspace decomposition to vary the boundary between frozencore and active orbitals and found that freezing up to $3 p$ leads to the disappearance of the $\mathrm{RH}$, which shows the essential role of the $3 p$ electrons. Then we have calculated the contribution of each transition between the initial orbitals to the harmonic spectra, which has indeed revealed that the $\mathrm{RH}$ is dominated by the $3 p-3 d(m=0, \pm 1)$ transitions, constructively interfering. It has followed from the inspection of the HHG timefrequency structure that the RH is emitted mainly, if not exclusively, when the sum of the kinetic energy of the returning electron and the ionization potential of $\mathrm{Mn}$ or $\mathrm{Mn}^{+}$is $\sim 50 \mathrm{eV}$. This implies that the electron recombines to the autoionizing upper state of the $3 p-3 d$ transitions, as proposed previously within the single-active-electron approximation using a model potential barrier [16,17,21,22].

\section{ACKNOWLEDGMENTS}

This research was supported in part by a Grant-in-Aid for Scientific Research (Grants No. 16H03881, No. 17K05070, No. 18H03891, and No. 19H00869) from the Ministry of Education, Culture, Sports, Science and Technology (MEXT) of Japan and also by the Photon Frontier Network Program of MEXT. This research was also partially supported by JST COI (Grant No. JPMJCE1313), JST CREST (Grant No. JPMJCR15N1), and by Quantum Leap Flagship Program of MEXT. I.S.W. gratefully acknowledges support from Special Graduate Program in Resilience Engineering of the University of Tokyo.
[1] D. Baykusheva, S. Brennecke, M. Lein, and H. J. Wörner, Phys. Rev. Lett. 119, 203201 (2017).

[2] M. F. Ciappina, J. A. Pérez-Hernández, A. S. Landsman, T. Zimmermann, M. Lewenstein, L. Roso, and F. Krausz, Phys. Rev. Lett. 114, 143902 (2015).

[3] L. Gui-Hua, X. Hong-Qiang, Y. Jin-Ping, C. Wei, C. Ya, L. Xiao-Jun, C. Jing, and X. Xin-Hua, Acta Phys. Sin-Ch. Ed. 65, 224208 (2016).
[4] M. C. H. Wong, A.-T. Le, A. F. Alharbi, A. E. Boguslavskiy, R. R. Lucchese, J.-P. Brichta, C. D. Lin, and V. R. Bhardwaj, Phys. Rev. Lett. 110, 033006 (2013).

[5] T. D. Scarborough, T. T. Gorman, F. Mauger, P. Sándor, S. Khatri, M. B. Gaarde, K. J. Schafer, P. Agostini, and L. F. DiMauro, Appl. Sci. 8, 1129 (2018).

[6] P. Agostini and L. F. DiMauro, Rep. Prog. Phys. 67, 1563 (2004) 
[7] F. Krausz and M. Ivanov, Rev. Mod. Phys. 81, 163 (2009).

[8] M. Protopapas, C. H. Keitel, and P. L. Knight, Rep. Prog. Phys. 60, 389 (1997).

[9] R. A. Ganeev, M. Suzuki, M. Baba, H. Kuroda, and T. Ozaki, Opt. Lett. 31, 1699 (2006).

[10] M. Suzuki, M. Baba, R. Ganeev, H. Kuroda, and T. Ozaki, Opt. Lett. 31, 3306 (2006).

[11] R. A. Ganeev, P. A. Naik, H. Singhal, J. A. Chakera, and P. D. Gupta, Opt. Lett. 32, 65 (2007).

[12] R. A. Ganeev, T. Witting, C. Hutchison, F. Frank, M. Tudorovskaya, M. Lein, W. A. Okell, A. Zaï, J. P. Marangos, and J. W. G. Tisch, Opt. Express 20, 25239 (2012).

[13] R. A. Ganeev, J. A. Chakera, P. A. Naik, H. Singhal, R. A. Khan, and P. D. Gupta, J. Opt. Soc. Am. B 28, 1055 (2011).

[14] R. A. Ganeev, V. V. Strelkov, C. Hutchison, A. Zaïr, D. Kilbane, M. A. Khokhlova, and J. P. Marangos, Phys. Rev. A 85, 023832 (2012).

[15] R. A. Ganeev, L. B. Elouga Bom, J.-C. Kieffer, and T. Ozaki, Phys. Rev. A 75, 063806 (2007).

[16] S. Haessler, V. Strelkov, L. B. E. Bom, M. Khokhlova, O. Gobert, J.-F. Hergott, F. Lepetit, M. Perdrix, T. Ozaki, and P. Salières, New J. Phys. 15, 013051 (2013).

[17] M. Fareed, V. Strelkov, N. Thiré, S. Mondal, B. Schmidt, F. Légaré, and T. Ozaki, Nat. Commun. 8, 16061 (2017).

[18] D. B. Milošević, J. Phys. B 40, 3367 (2007).

[19] D. B. Milošević, J. Opt. Soc. Am. B 23, 308 (2006).

[20] V. V. Strelkov, M. A. Khokhlova, and N. Y. Shubin, Phys. Rev. A 89, 053833 (2014).

[21] M. Tudorovskaya and M. Lein, Phys. Rev. A 84, 013430 (2011).

[22] M. V. Frolov, N. L. Manakov, and A. F. Starace, Phys. Rev. A 82, 023424 (2010).

[23] D. B. Milošević, Phys. Rev. A 81, 023802 (2010).

[24] V. Strelkov, Phys. Rev. Lett. 104, 123901 (2010).

[25] I. A. Ivanov and A. S. Kheifets, Phys. Rev. A 78, 053406 (2008).

[26] C. F. de Morisson Faria, R. Kopold, W. Becker, and J. M. Rost, Phys. Rev. A 65, 023404 (2002).

[27] P. V. Redkin and R. A. Ganeev, Phys. Rev. A 81, 063825 (2010).

[28] S. Pabst and R. Santra, Phys. Rev. Lett. 111, 233005 (2013).

[29] L. Greenman, P. J. Ho, S. Pabst, E. Kamarchik, D. A. Mazziotti, and R. Santra, Phys. Rev. A 82, 023406 (2010).

[30] K. L. Ishikawa and T. Sato, IEEE J. Sel. Top. Quantum Electron. 21, 1 (2015).

[31] T. Sato and K. L. Ishikawa, Phys. Rev. A 88, 023402 (2013).

[32] T. Sato, K. L. Ishikawa, I. Březinová, F. Lackner, S. Nagele, and J. Burgdörfer, Phys. Rev. A 94, 023405 (2016).
[33] Y. Orimo, T. Sato, A. Scrinzi, and K. L. Ishikawa, Phys. Rev. A 97, 023423 (2018).

[34] T. Sato, Y. Orimo, T. Teramura, O. Tugs, and K. L. Ishikawa, in Progress in Ultrafast Intense Laser Science XIV, edited by K. Yamanouchi, P. Martin, M. Sentis, L. Ruxin, and D. Normand (Springer, Switzerland, 2018), p. 143.

[35] T. Sato and K. L. Ishikawa, Phys. Rev. A 91, 023417 (2015).

[36] I. Tikhomirov, T. Sato, and K. L. Ishikawa, Phys. Rev. Lett. 118, 203202 (2017).

[37] Y. Li, T. Sato, and K. L. Ishikawa, Phys. Rev. A 99, 043401 (2019).

[38] F. A. Ilkov, J. E. Decker, and S. L. Chin, J. Phys. B 25, 4005 (1992).

[39] C. Corliss and J. Sugar, J. Phys. Chem. Ref. Data 6, 1253 (1977).

[40] P. G. Reinhard, Z. Phys. A 280, 281 (1977).

[41] P.-O. Löwdin and P. Mukherjee, Chem. Phys. Lett. 14, 1 (1972)

[42] R. Moccia, Int. J. Quantum Chem. 7, 779 (1973).

[43] E. J. Heller, J. Chem. Phys. 64, 63 (1976).

[44] T. N. Rescigno and C. W. McCurdy, Phys. Rev. A 62, 032706 (2000).

[45] C. W. McCurdy, M. Baertschy, and T. N. Rescigno, J. Phys. B 37, R137 (2004).

[46] B. I. Schneider, L. A. Collins, and S. X. Hu, Phys. Rev. E 73, 036708 (2006).

[47] B. I. Schneider, J. Feist, S. Nagele, R. Pazourek, S. X. Hu, L. A. Collins, and J. Burgdörfer, in Quantum Dynamic Imaging, edited by A. D. Bandrauk and M. Ivanov (Springer, New York, 2011), p. 149.

[48] A. Scrinzi, Phys. Rev. A 81, 053845 (2010).

[49] T. Koga, S. Watanabe, K. Kanayama, R. Yasuda, and A. J. Thakkar, J. Chem. Phys. 103, 3000 (1995).

[50] P. B. Corkum, Phys. Rev. Lett. 71, 1994 (1993).

[51] K. C. Kulander, K. J. Schafer, and J. L. Krause, in Super-Intense Laser-Atom Physics, edited by A. L'Huillier, B. Piraux, and K. Rzazewski (Springer, New York, 1993), p. 95.

[52] J. W. Cooper, C. W. Clark, C. L. Cromer, T. B. Lucatorto, B. F. Sonntag, E. T. Kennedy, and J. T. Costello, Phys. Rev. A 39, 6074 (1989).

[53] A. von dem Borne, R. L. Johnson, B. Sonntag, M. Talkenberg, A. Verweyen, Ph. Wernet, J. Schulz, K. Tiedtke, Ch. Gerth, B. Obst, P. Zimmermann, and J. E. Hansen, Phys. Rev. A 62, 052703 (2000).

[54] P. M. Abanador, F. Mauger, K. Lopata, M. B. Gaarde, and K. J. Schafer, Phys. Rev. A 97, 043414 (2018). 